\begin{tabular}{|c|c|c|c|}
\hline TITLE: & \multicolumn{3}{|c|}{$\begin{array}{l}\text { TEMPERATURE DISTRIBUTION IN A FLOWING FLUID } \\
\text { HEATED IN A MICROWAVE RESONANT CAVITY }\end{array}$} \\
\hline AUTHOR(S): & $\begin{array}{l}\text { Eric M. Nelson } \\
\text { Ray M. Stringfield } \\
\text { Robert J. Kares } \\
\text { J. R. Thomas, Jr. }\end{array}$ & $\begin{array}{l}\text { AOT-9 } \\
\text { AOT-9 } \\
\text { X-PA } \\
\text { AOT-9 }\end{array}$ & $\begin{array}{l}\text { PECWEIVE } \\
181995\end{array}$ \\
\hline
\end{tabular}

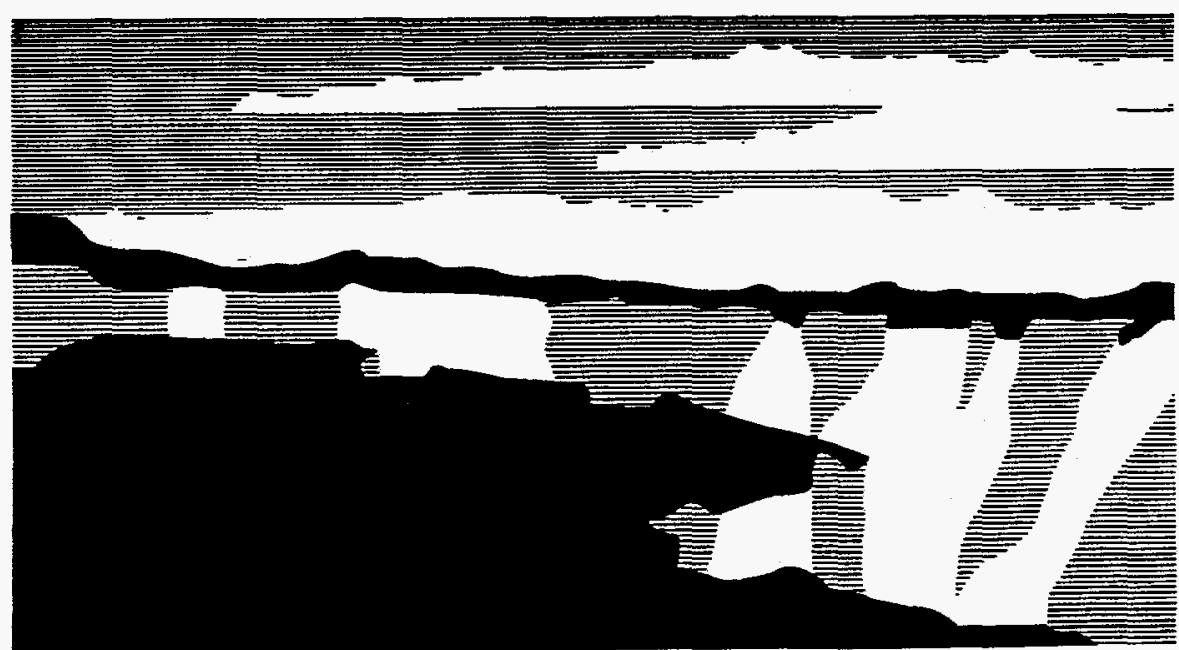

NATIONAL LABORATORY

Los Alamos National Laboratory, an affirmative action/equal opportunity employer, is operated by the University of California for the U.S. Department of Energy under contract W-7405-ENG-36. By acceptance of this article, the publisher recognizes that the U.S. Government retains a nonexclusive, royalty-free license to publish or reproduce the published form of this contribution, or to allow others to do so, for U.S. Government purposes. The Los Alamos National Laboratory requests that the publisher identify this article as work performed under the auspices of the U.S. Department of Energy. 


\title{
TEMPERATURE DISTRIBUTION IN A FLOWING FLUID HEATED IN A MICROWAVE RESONANT CAVITY
}

\author{
J.R. THOMAS, JR!, ERIC M. NELSON‡ ROBERT J. KARES‡ RAY M. STRINGFIELD \\ $\dagger$ Mechanical Engineering Department, \\ Virginia Polytechnic Institute and State University, Blacksburg, VA 24061 \\ $\ddagger$ Los Alamos National Laboratory, Los Alamos, NM 87545
}

\begin{abstract}
This paper presents results of an analytical study of microwave heating of a fluid flowing through a tube situated along the axis of a cylindrical microwave applicator. The interaction of the microwave field pattern and the fluid velocity profiles is illustrated for both laminar and turbulent flow. Resulting temperature profiles are compared with those generated by conventional heating through a surface heat flux. It is found that microwave heating offers several advantages over conventional heating.
\end{abstract}

\section{INTRODUCTION}

A logical industrial application for microwave energy is that of heating a process fluid. This can be accomplished by passing the fluid through a microwave applicator at an appropriate rate to heat the fluid to the desired temperature. For many process objectives, the temperature distribution in the fluid would be of primary interest. The temperature distribution in the fluid depends on both the velocity profile of the flowing fluid and the distribution of electromagnetic field strength in the cavity. In the present work, we consider a lossy fluid flowing through a cylindrical resonant cavity and show that the presence of the fluid strongly influences the field distribution, but that the fluid temperature distribution is more sensitive to the flow regime than to the shape of the electromagnetic field. Microwave heating of flowing fluids is shown to have significant advantages over conventional heating if uniform fluid temperatures are desired.

\section{ELECTROMAGNETIC FIELD DISTRIBUTION}

A right circular cylindrical resonant cavity is a logical choice for the applicator. Typically, such a cavity would be excited in the $\mathrm{TM}_{010}$ mode to heat a cylinder of product on the axis. The presence of the load causes the driven fields to differ significantly from a single mode pattern, however.

The field calculation procedure has been described in detail elsewhere [1], and will be only briefly summarized. The system is modeled as a right circular cylinder consisting of two regions: load and air. The product is assumed to have an hypothetical complex permittivity $\epsilon=10+2 i$. The microwave source is a small aperture at the midplane of the applicator fed by a rectangular waveguide. The field is determined as a superposition of modes, all of which have the form of Bessel functions of complex argument. We considered a cavity of diameter $D=10.86 \mathrm{~cm}$, height $L=62.59 \mathrm{~cm}$, with a cylindrical tube on the axis having diameter $2 r_{0}=2.086 \mathrm{~cm}$. It was determined that a total of 34 modes are necessary to adequately represent the field distribution in the load. The resulting field pattern on an $r-z$ slice through the drive plane is compared to the pure $\mathrm{TM}_{010}$ mode in Fig. 1 . It is apparent that the field is concentrated to one side of the load centerline, opposite the feed aperture. Clearly this would lead to a hot spot in a stationary product. Below, we consider the effect of this field on an electromagnetically lossy flowing fluid. 


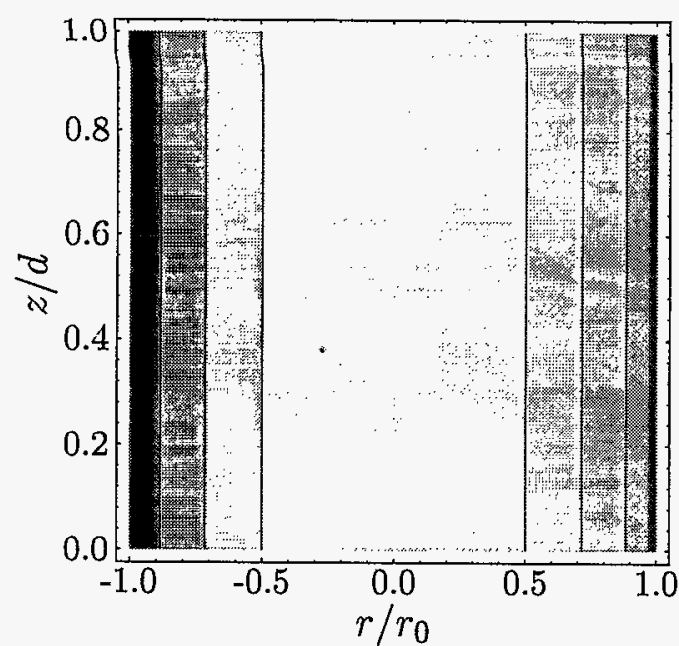

(a)
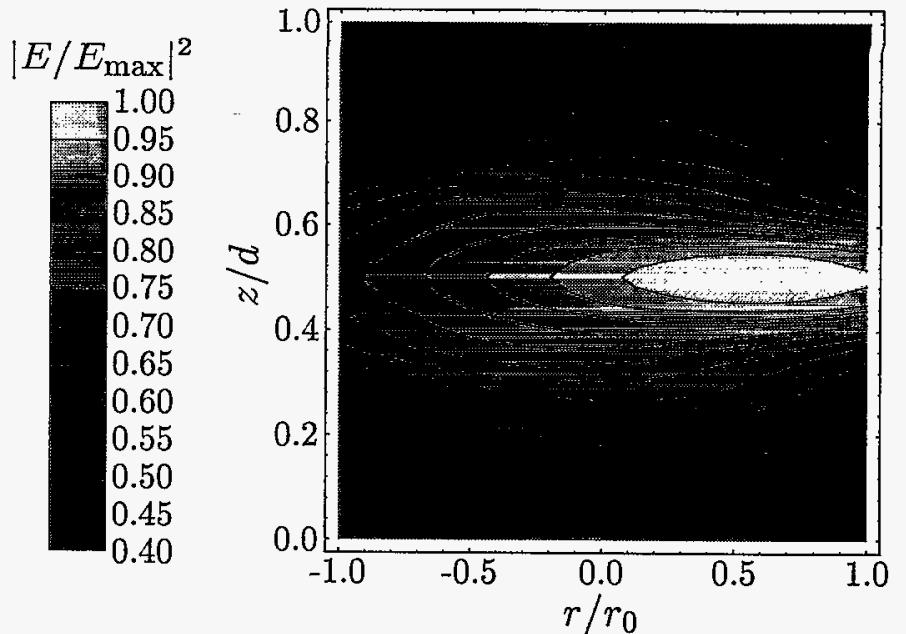

(b)

Figure 1. Electric field distribution in the tube portion of the microwave applicator. Plot (a) shows a pure $\mathrm{TM}_{010}$ mode. Plot (b) is a more realistic picture of the field driven by an aperture at the midplane on the cavity wall (off of plot to right).

\section{TEMPERATURE DISTRIBUTION}

We assume steady flow and steady-state conditions for a fluid flowing through a cylindrical tube on the axis of the cylindrical cavity described above. Temperature distributions were computed using the thermal properties of water for both laminar and turbulent flow. The equations describing the velocity and temperature distributions are

$$
\frac{1}{\rho} \frac{d P}{d z}=\frac{1}{r} \frac{\partial}{\partial r}\left[r\left(\nu+\epsilon_{M}\right) \frac{\partial u}{\partial r}\right]+\frac{1}{r} \frac{\partial}{\partial \theta}\left[\frac{1}{r}\left(\nu+\epsilon_{M}\right) \frac{\partial u}{\partial \theta}\right] ;
$$

and

$$
u \frac{\partial T}{\partial z}=\frac{\dot{q}}{\rho C_{p}}+\frac{1}{r} \frac{\partial}{\partial r}\left[r\left(\alpha+\epsilon_{H}\right) \frac{\partial T}{\partial r}\right]+\frac{1}{r^{2}} \frac{\partial}{\partial \theta}\left[r\left(\alpha+\epsilon_{H}\right) \frac{\partial T}{\partial \theta}\right] .
$$

Eqs. (1) and (2) represent conservation of momentum and energy of the fluid, respectively. In these equations, $P$ represents the pressure, $\rho$ the density, and $u$ the local fluid velocity parallel to the $z$-axis. Also, $T$ is the local temperature, $C_{p}$ the specific heat at constant pressure, and $\dot{q}$ represents the local energy generation density caused by absorption of microwave energy. Finally, $\nu$ represents the kinematic viscosity and $\alpha$ the thermal diffusivity; $\epsilon_{M}$ and $\epsilon_{H}$ are turbulence parameters.

These equations were solved by a finite-difference technique, using literature data for the temperature dependence of all physical properties. Ordinary water was used as the working fluid in these calculations. For values of Reynolds number $R e=\rho u_{m} D / \mu<2300$, the flow was assumed to be laminar, and $\epsilon_{M}$ and $\epsilon_{H}$ were set to zero. For turbulent flow, these parameters were determined according to the Prandtl-Von Karmann turbulence model [2].

Typical velocity profiles for laminar and turbulent flow are shown in Fig. 2. These profiles differ from the standard shapes shown in textbooks because of the inclusion of temperature-dependent viscosity and volumetric heating of the fluid. Nevertheless, laminar flow displays a much more peaked distribution than turbulent flow, where mixing smooths the velocity distribution. We would expect these velocity distributions to strongly affect temperature distributions resulting from fluid heating. Clearly the fluid in the center of the tube, because of its higher velocity, will have less time to be heated by a microwave field. 


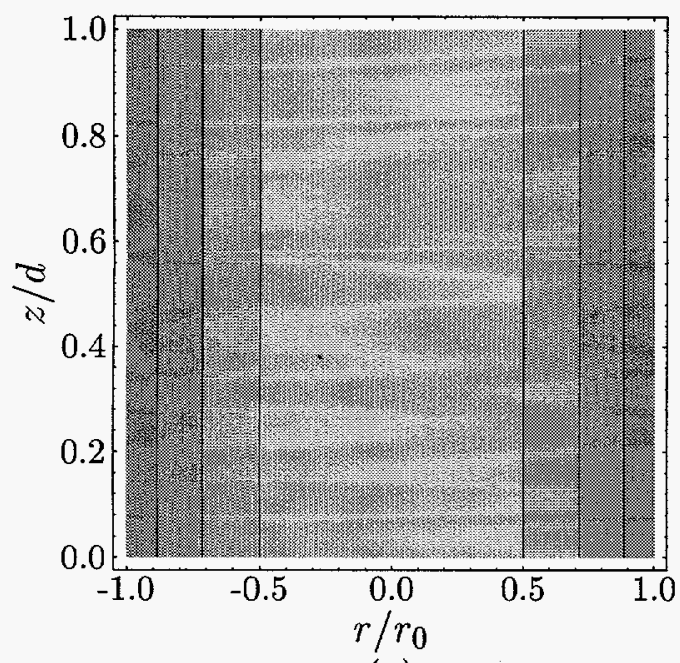

(a)

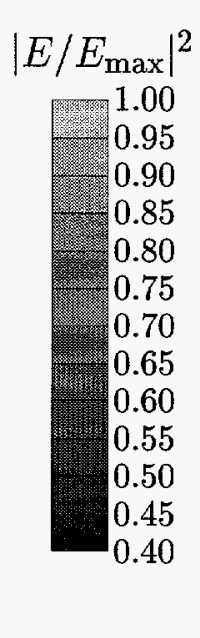

(a)

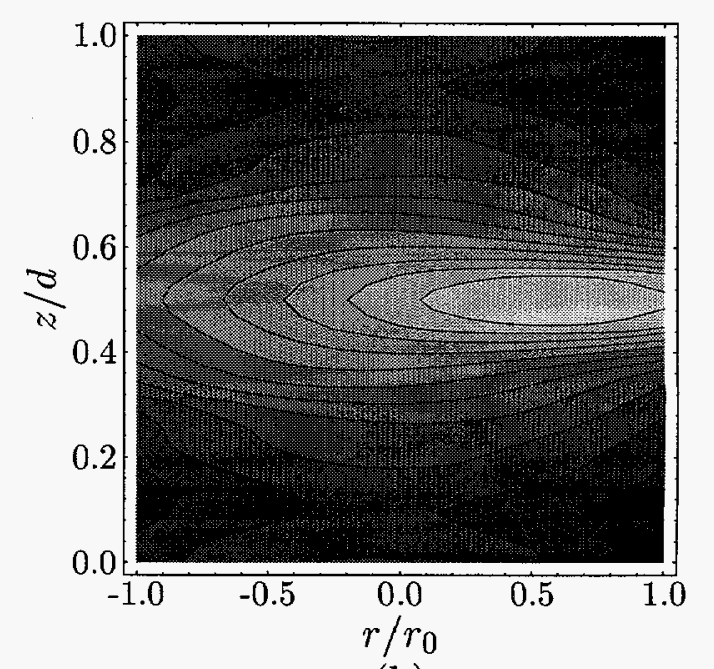

(b)

Figure 1. Electric field distribution in the tube portion of the microwave applicator. Plot (a) shows a pure $\mathrm{TM}_{010}$ mode. Plot (b) is a more realistic picture of the field driven by an aperture at the midplane on the cavity wall (off of plot to right).

\section{TEMPERATURE DISTRIBUTION}

We assume steady flow and steady-state conditions for a fluid flowing through a cylindrical tube on the axis of the cylindrical cavity described above. Temperature distributions were computed using the thermal properties of water for both laminar and turbulent flow. The equations describing the velocity and temperature distributions are

$$
\frac{1}{\rho} \frac{d P}{d z}=\frac{1}{r} \frac{\partial}{\partial r}\left[r\left(\nu+\epsilon_{M}\right) \frac{\partial u}{\partial r}\right]+\frac{1}{r} \frac{\partial}{\partial \theta}\left[\frac{1}{r}\left(\nu+\epsilon_{M}\right) \frac{\partial u}{\partial \theta}\right] ;
$$

and

$$
u \frac{\partial T}{\partial z}=\frac{\dot{q}}{\rho C_{p}}+\frac{1}{r} \frac{\partial}{\partial r}\left[r\left(\alpha+\epsilon_{H}\right) \frac{\partial T}{\partial r}\right]+\frac{1}{r^{2}} \frac{\partial}{\partial \theta}\left[r\left(\alpha+\epsilon_{H}\right) \frac{\partial T}{\partial \theta}\right] .
$$

Eqs. (1) and (2) represent conservation of momentum and energy of the fluid, respectively. In these equations, $P$ represents the pressure, $\rho$ the density, and $u$ the local fluid velocity parallel to the $z$-axis. Also, $T$ is the local temperature, $C_{p}$ the specific heat at constant pressure, and $\dot{q}$ represents the local energy generation density caused by absorption of microwave energy. Finally, $\nu$ represents the kinematic viscosity and $\alpha$ the thermal diffusivity; $\epsilon_{M}$ and $\epsilon_{H}$ are turbulence parameters.

These equations were solved by a finite-difference technique, using literature data for the temperature dependence of all physical properties. Ordinary water was used as the working fluid in these calculations. For values of Reynolds number $R e=\rho u_{m} D / \mu<2300$, the flow was assumed to be laminar, and $\epsilon_{M}$ and $\epsilon_{H}$ were set to zero. For turbulent flow, these parameters were determined according to the Prandtl-Von Karmann turbulence model [2].

Typical velocity profiles for laminar and turbulent flow are shown in Fig. 2. These profiles differ from the standard shapes shown in textbooks because of the inclusion of temperature-dependent viscosity and volumetric heating of the fluid. Nevertheless, laminar flow displays a much more peaked distribution than turbulent flow, where mixing smooths the velocity distribution. We would expect these velocity distributions to strongly affect temperature distributions resulting from fluid heating. Clearly the fluid in the center of the tube, because of its higher velocity, will have less time to be heated by a microwave field. 


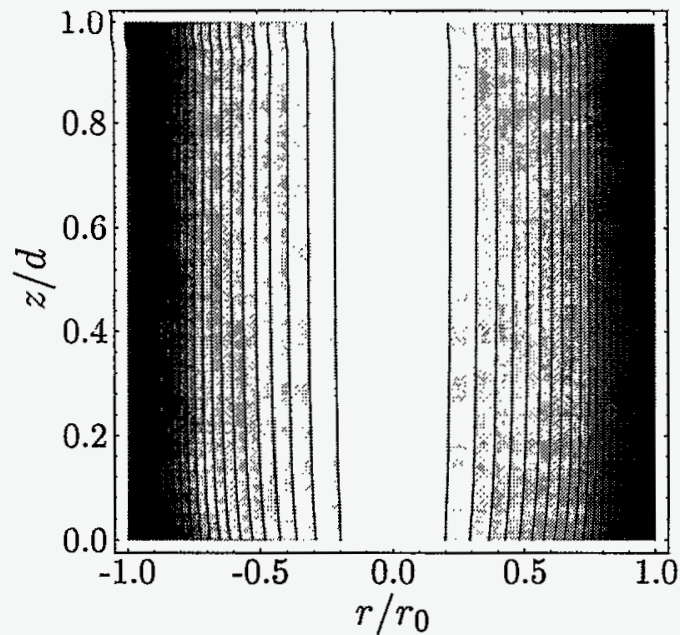

(a)

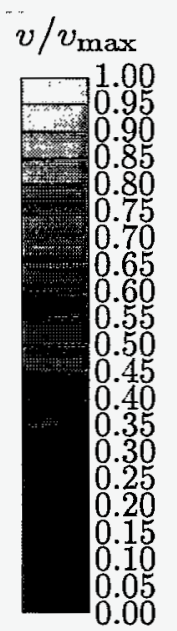

Figure 2. Normalized velocity profiles for (a) laminar flow and (b) turbulent flow. The velocity distribution is more uniform for the turbulent case, which leads to the more uniform temperature distributions shown below.

Thus for a uniform field in the tube we would expect the temperature profile to approximate the inverse of the velocity profile.

The $\mathrm{TM}_{010}$ mode produces a nearly uniform field across the tube cross section, leading to the temperature distributions shown on a $r$ - $z$ slice through the drive plane in Fig. 3(a) and Fig. 3(b). This field pattern produces a $30^{\circ} \mathrm{C}$ temperature difference between fluid in the center of the tube and that near the tube wall in laminar flow. Such a temperature distribution would be very undesirable for many process applications. Turbulent flow, however, produces very uniform temperatures as is apparent in Fig. 3(b).

Similar results for the realistic field pattern of Fig. 1(b) are shown in Fig. 3(c) and Fig. 3(d). Surprisingly, the rather nonuniform field pattern has little effect on the fluid temperature distribution. The field concentration seen in Fig. 1(b) is reflected in the fluid temperatures as a slight constriction in the temperature contours on the right side of the figure. Thus it appears that local nonuniformities in the microwave field are greatly overshadowed by the effects of the flow regime in determining the temperature profile in a flowing fluid heated by microwaves.

\section{CONVENTIONAL HEATING}

To provide a basis for comparison, fluid temperature profiles were computed for conventional heating of water flowing in a tube of identical size and fluid mass flow rate as that considered for microwave heating. The conventional heating was provided by a uniform surface heat flux applied at the tube surface of an appropriate magnitude to produce an exit temperature just below $100^{\circ} \mathrm{C}$. The temperature distributions are shown in Fig. 3(e) for laminar flow and Fig. 3(f) for turbulent flow. In these figures, darker areas are cooler; it is seen that for laminar flow (worst case), the entire center of the flow is still at the entry temperature at the exit. In fact, the velocity-weighted mean temperature at the exit is only $32.1^{\circ} \mathrm{C}$, while the fluid near the wall has reached $87.3^{\circ} \mathrm{C}$. To heat the flow uniformly to $87^{\circ} \mathrm{C}$ would require a very long tube and a much smaller surface heat flux. Comparing this with Fig. 3(c) reveals the distinct advantage of microwave heating. 


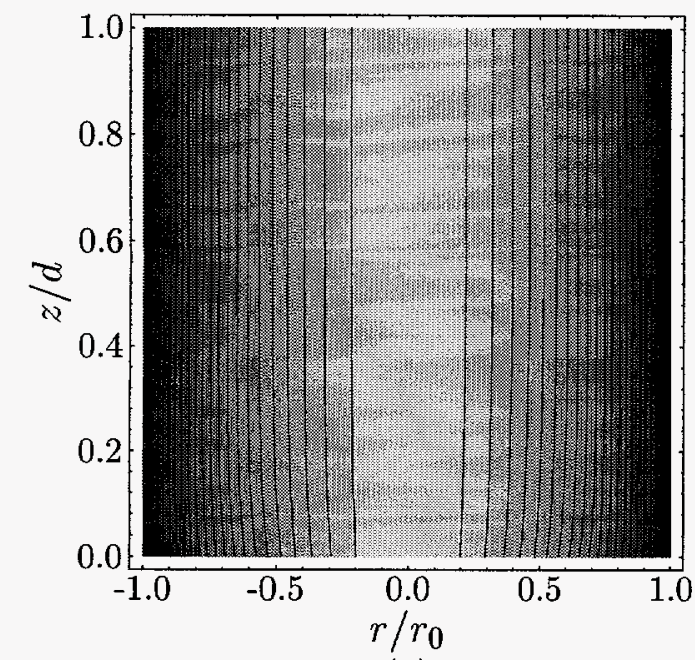

(a)

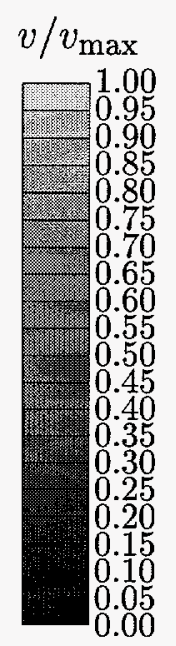

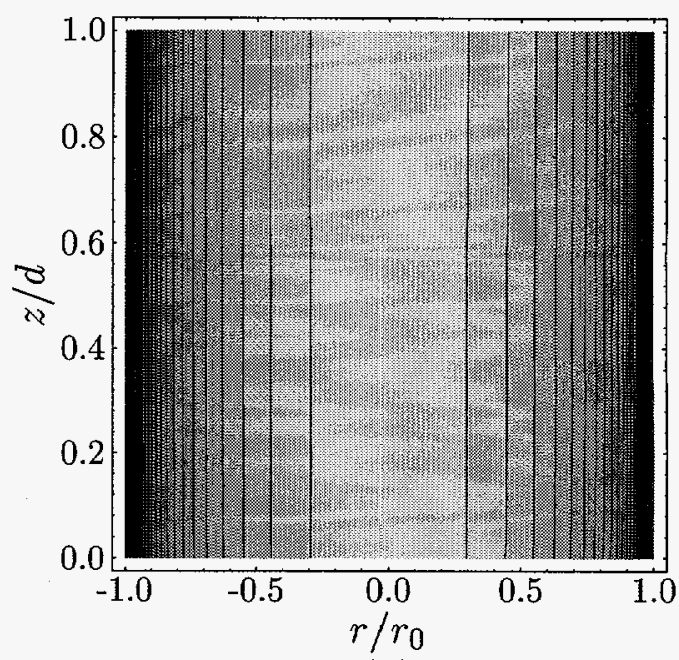

(b)

Figure 2. Normalized velocity profiles for (a) laminar flow and (b) turbulent flow. The velocity distribution is more uniform for the turbulent case, which leads to the more uniform temperature distributions shown below.

Thus for a uniform field in the tube we would expect the temperature profile to approximate the inverse of the velocity profile.

The $\mathrm{TM}_{010}$ mode produces a nearly uniform field across the tube cross section, leading to the temperature distributions shown on a $r-z$ slice through the drive plane in Fig. 3(a) and Fig. 3(b). This field pattern produces a $30^{\circ} \mathrm{C}$ temperature difference between fluid in the center of the tube and that near the tube wall in laminar flow. Such a temperature distribution would be very undesirable for many process applications. Turbulent flow, however, produces very uniform temperatures as is apparent in Fig. 3(b).

Similar results for the realistic field pattern of Fig. 1(b) are shown in Fig. 3(c) and Fig. 3(d). Surprisingly, the rather nonuniform field pattern has little effect on the fluid temperature distribution. The field concentration seen in Fig. 1(b) is reflected in the fluid temperatures as a slight constriction in the temperature contours on the right side of the figure. Thus it appears that local nonuniformities in the microwave field are greatly overshadowed by the effects of the flow regime in determining the temperature profile in a flowing fluid heated by microwaves.

\section{CONVENTIONAL HEATING}

To provide a basis for comparison, fluid temperature profiles were computed for conventional heating of water flowing in a tube of identical size and fluid mass flow rate as that considered for microwave heating. The conventional heating was provided by a uniform surface heat flux applied at the tube surface of an appropriate magnitude to produce an exit temperature just below $100^{\circ} \mathrm{C}$. The temperature distributions are shown in Fig. 3(e) for laminar flow and Fig. 3(f) for turbulent flow. In these figures, darker areas are cooler; it is seen that for laminar flow (worst case), the entire center of the flow is still at the entry temperature at the exit. In fact, the velocity-weighted mean temperature at the exit is only $32.1^{\circ} \mathrm{C}$, while the fluid near the wall has reached $87.3^{\circ} \mathrm{C}$. To heat the flow uniformly to $87^{\circ} \mathrm{C}$ would require a very long tube and a much smaller surface heat flux. Comparing this with Fig. 3(c) reveals the distinct advantage of microwave heating. 


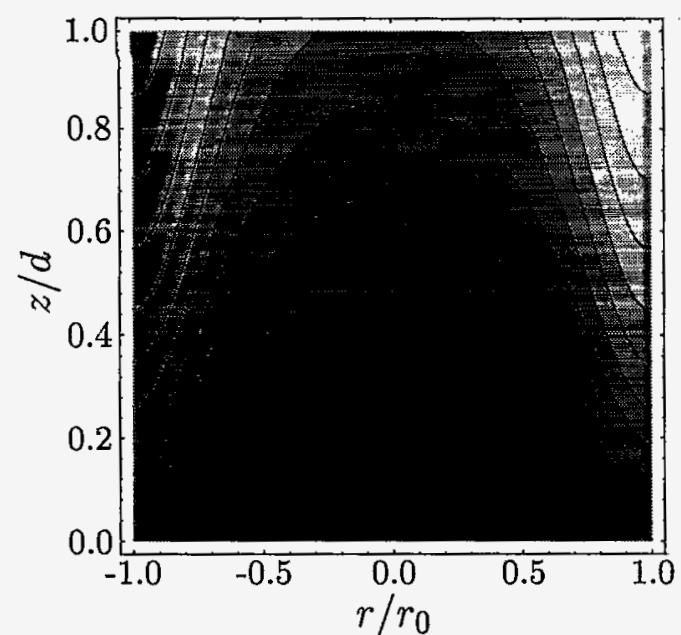

(a)

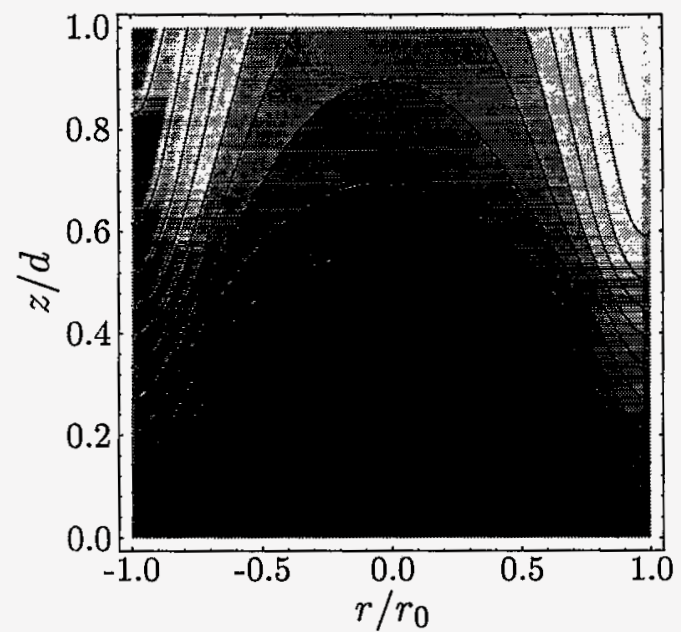

(c)

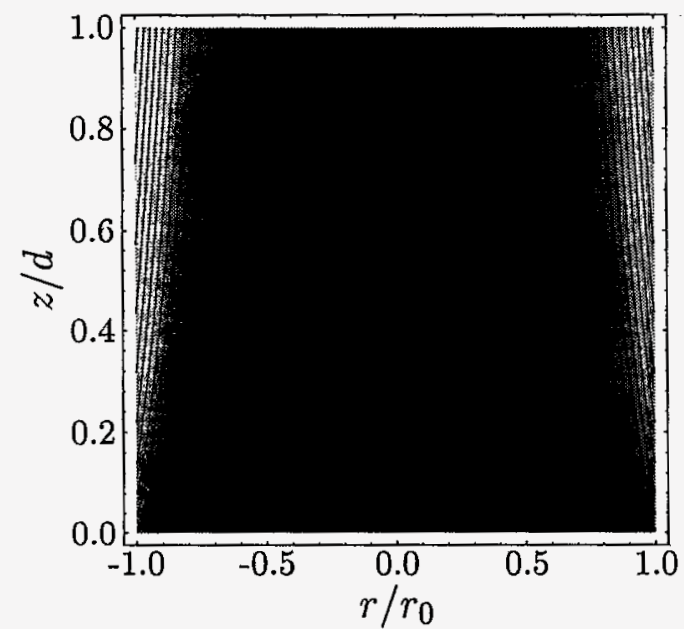

(e)
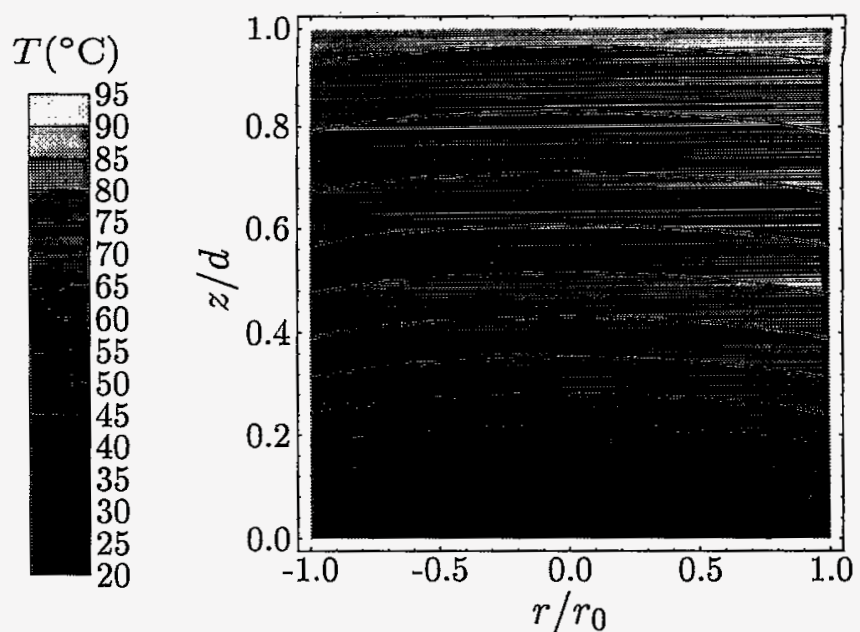

(b)

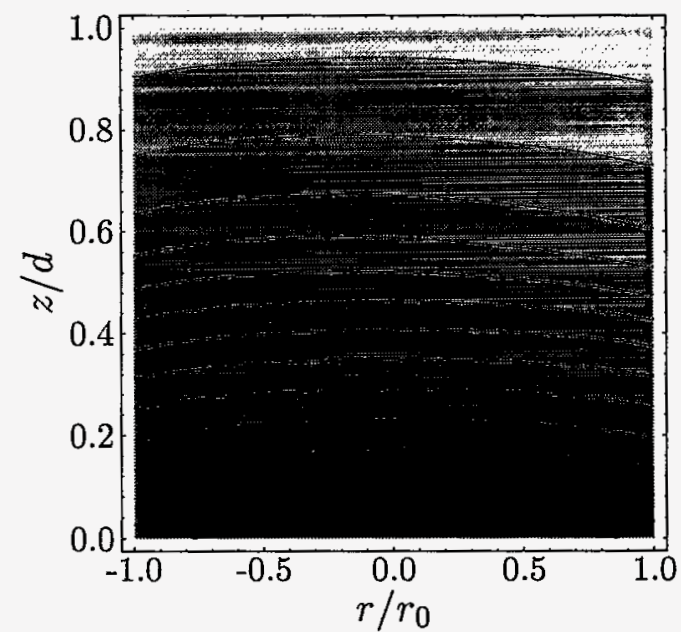

(d)

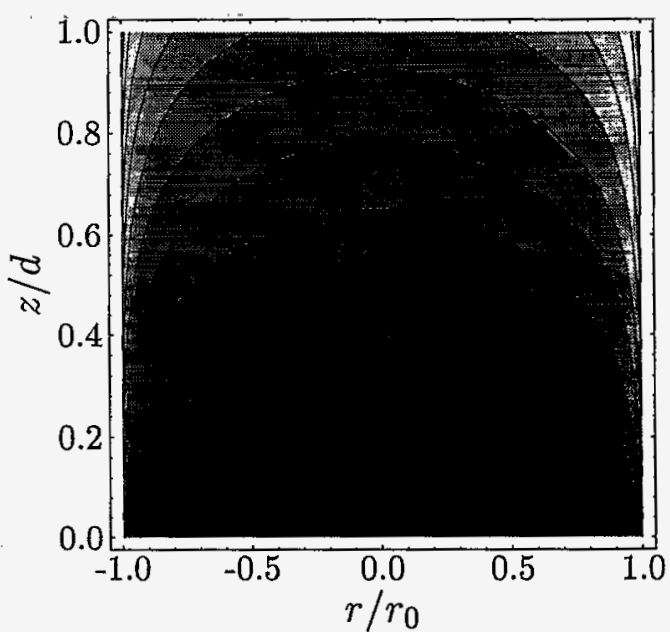

(f)

Figure 3. Product temperature on an $r-z$ slice. The top two cases, (a) and (b), are heated by a pure $\mathrm{TM}_{010}$ mode field. The middle two cases, (c) and (d), are heated by a more realistic electric field which is driven by an aperture at the cavity wall to the right of the plot. The bottom two cases, (e) and (f), are conventionally heated. The cases on the left, (a), (c) and (e), are for laminar flow. The cases on the right, (b), (d) and (f), are for turbulent flow. 


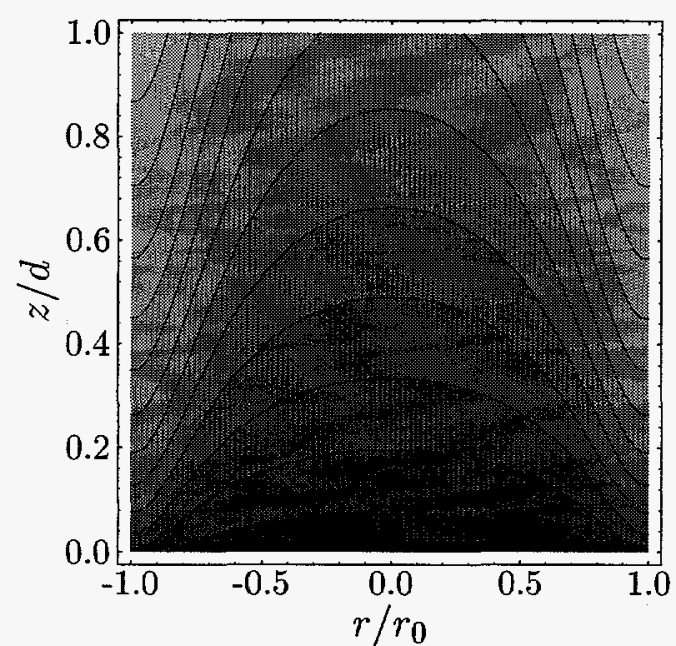

(a)

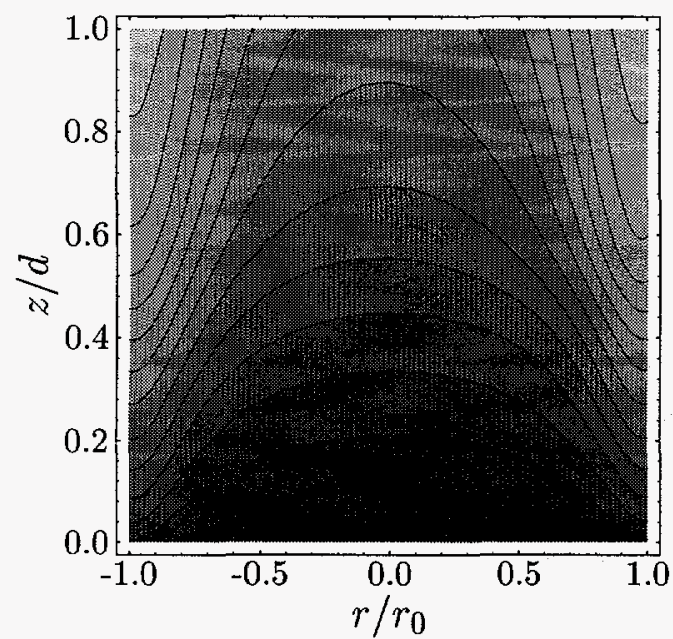

(c)

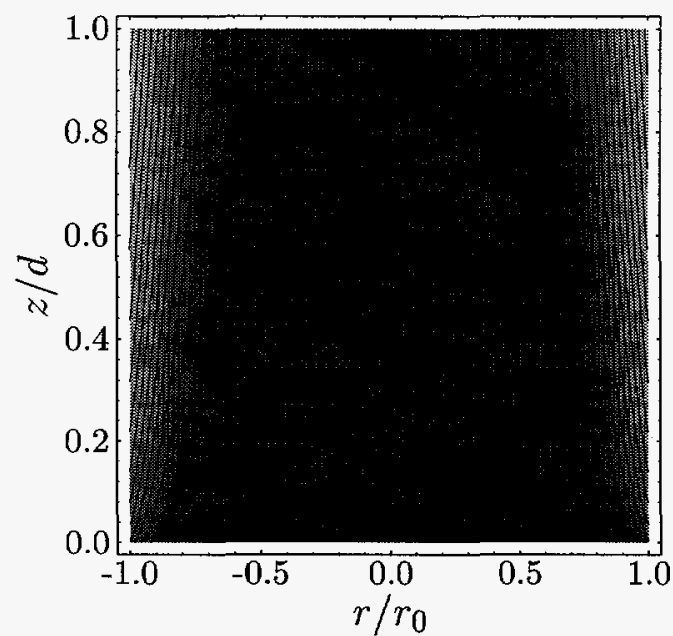

(e)
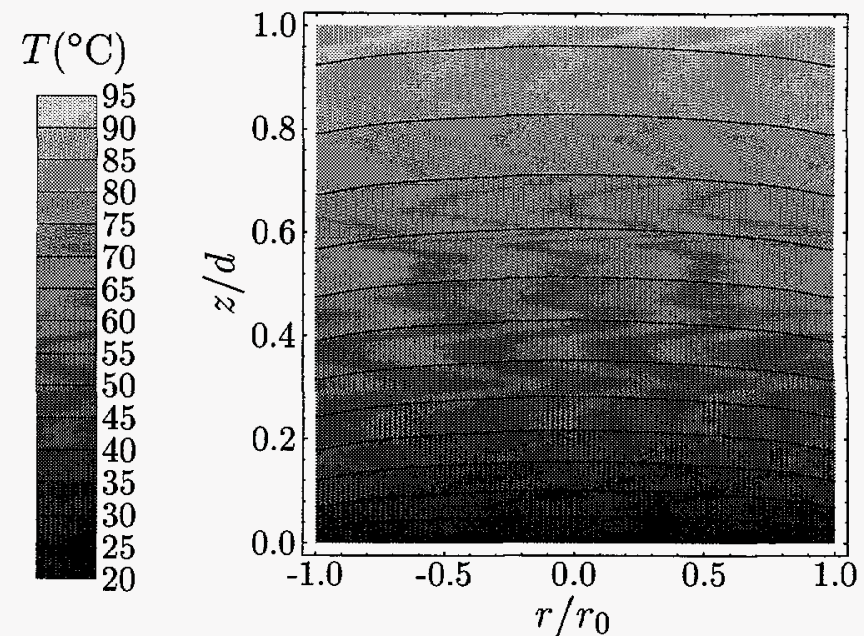

(b)

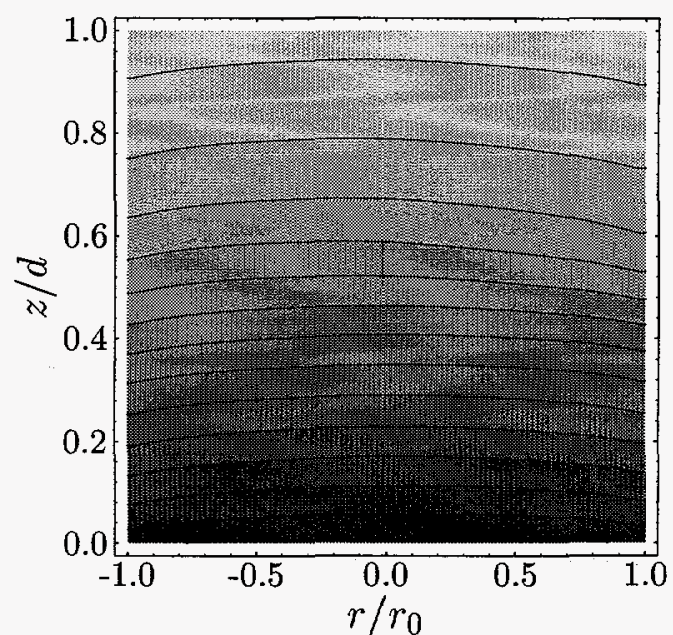

(d)

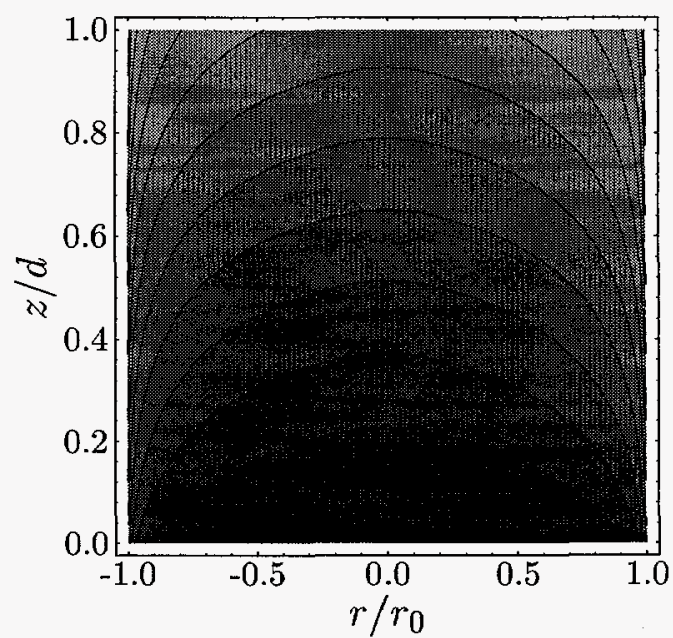

(f)

Figure 3. Product temperature on an $r-z$ slice. The top two cases, (a) and (b), are heated by a pure $\mathrm{TM}_{010}$ mode field. The middle two cases, (c) and (d), are heated by a more realistic electric field which is driven by an aperture at the cavity wall to the right of the plot. The bottom two cases, (e) and (f), are conventionally heated. The cases on the left, (a), (c) and (e), are for laminar flow. The cases on the right, (b), (d) and (f), are for turbulent flow. 


\section{- CONCLUSIONS}

A cylindrical resonant cavity may be used as an applicator for heating a flowing fluid. The presence of the lossy fluid significantly alters the field distribution in the cavity from the design mode based on an empty cavity. However, the flow regime of the fluid is much more important in determining the uniformity of the temperature distribution in the fluid, with turbulent flow being greatly preferred if uniform temperatures are desired. Microwave heating in a properly designed applicator can produce much more uniform fluid temperatures than conventional heating.

\section{REFERENCES}

1. Nelson, E. M., R. J. Kares, and R. M. Stringfield, "Semi-Analytic Computation of the Driven Fields in Right Circular Cylinder Microwave Applicators", Proceedings, 30th Microwave Power Symposium, 1995.

2. Bejan, A., Convection Heat Transfer, John Wiley \& Sons, New York, 1984. 


\section{DISCLAIMER}

This report was prepared as an account of work sponsored by an agency of the United States Government. Neither the United States Government nor any agency thereof, nor any of their employees, makes any warranty, express or implied, or assumes any legal liability or responsibility for the accuracy, completeness, or usefulness of any information, apparatus, product, or process disclosed, or represents that its use would not infringe privately owned rights. Reference herein to any specific commercial product, process, or service by trade name, trademark, manufacturer, or otherwise does not necessarily constitute or imply its endorsement, recommendation, or favoring by the United States Government or any agency thereof. The views and opinions of authors expressed herein do not necessarily state or reflect those of the United States Government or any agency thereof. 